\title{
Physics Based Fatigue Compact Model for Ferroelectric Capacitors
}

\author{
Elmar Gondro ${ }^{1}$, Christian Kühn ${ }^{1}$, Franz Schuler ${ }^{2}$, and Oskar Kowarik ${ }^{1}$ \\ ${ }^{1}$ University of Bundeswehr, Institute of Electronics, ET 4, \\ Werner-Heisenberg-Weg 39, D-85577 Neubiberg, Germany \\ Elmar.Gondro@UniBw-Muenchen.DE, phone: +4989 6004-3662, fax: +49 89 6004-2223 \\ ${ }^{2}$ Infineon Technologies AG, Balanstraße 73, D-81541 München, Germany
}

\begin{abstract}
A physics based compact model describing the fatigue behavior of ferroelectric capacitors has been developed. Fatigue is a gradual decrease of detectable polarization with increasing number of polarization cycles. This can be caused by trapped charges which pin dipoles near the interface to the electrode. In order to polarize those pinned dipoles they have to be separated from the trapped charges by a higher electrical force. This force has been described in our model by additional coercive voltages representing the different polarization response of the dipoles in the interface region in contrast to those in the inner region of the ferroelectric capacitor. Our model has been implemented into a common-used circuit simulator showing good agreement with measurements.
\end{abstract}

\section{INTRODUCTION}

Ferroelectrics are widely investigated since they can be applied in low-energy-consuming, high-speed and radiation-hard non volatile semiconductor memories. Two important types of ferroelectric materials, namely lead zirconate titanate (PZT) and strontium bismuth tantalate (SBT) with a layered perovskite structure have been widely explored [1]. Specifically PZT is an attractive well-studied system, which has excellent ferroelectric properties and can be processed at relatively low temperatures $\left(550-600^{\circ} \mathrm{C}\right)$. However, PZT compounds with platinum electrodes exhibit significant polarization fatigue. Capacitors of this PZT compound fatigue rapidly [2] losing most of their remanent polarization after less than $10^{10}$ switching cycles. This is an essential limitation for the application in memories.

The fatigue can be reduced by the use of metaloxide electrodes [3] or layered perovskite ferroelectrics 4]. However, their switching polarization is small compared to PZT, and the control of electrical properties such as leakage current is not easy. Further, the processing temperature is high.

Another way to reduce fatigue characteristic is the use of low-field fatigue cycles, e.g. $2.5 \mathrm{~V}, 4000 \AA$, PZT [5], which is the relevant supply voltage in FRAM design.

Many kinds of interpretation for fatigue in ferro- electrics have been suggested, such as large-scale defect migration, domain pinning by defects or grain boundaries, and the screening at electrode interface (fig.11).

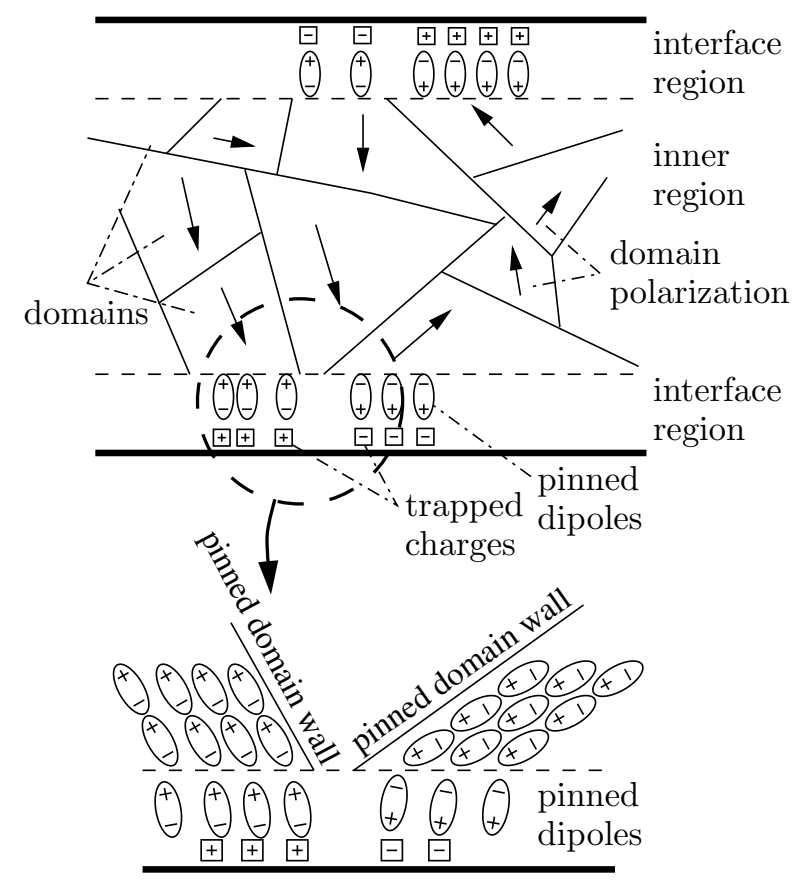

Fig. 1: Schematic of dipole and domain wall pinning.

However the fatigue mechanism is not yet clearly understood [6, 7, 8, 9].

For the design of memory circuits it is essential that we can simulate the fatigue behavior of the memory cells in the circuit. In this paper we present a compact model for circuit simulation. It is an expansion of the Preisach model, that includes fatigue effects.

\section{The original Preisach MOdel}

The basic idea of the Preisach model is that the ferroelectric material consists of a set of individual dipoles that contribute to the total polarization. Each of these dipoles has two individual coercive voltages, $v_{c}{ }^{+}$and $v_{c}{ }^{-}$, that are required to make it switch in the opposite direction. Thus each dipole has a rectangular hysteresis loop. Based on the assumption that the dipoles are non-interacting the hysteresis loop of 
the macroscopic system is seen as a superposition of these hysteresis units. $v_{c}{ }^{+}$and $v_{c}{ }^{-}$of the hysteresis units in the macroscopic system are statistically distributed. The distribution of the dipoles to their up and down states is dependent on the applied voltage and the course it has taken. This means that the turning points of the voltage curve are of great importance for the dipole distribution [10]. The total polarization $P$ of the ferroelectric results in:

$$
\begin{aligned}
& P\left(V, V_{1}, V_{2}, \ldots\right)= \\
& \int_{v_{c}+} \int_{v_{c}-} \rho\left(v_{c}{ }^{+}, v_{c}{ }^{-}\right) D\left(V, V_{1}, V_{2}, \ldots\right) p \mathrm{~d} v_{c}{ }^{-} \mathrm{d} v_{c}{ }^{+}
\end{aligned}
$$

$p$ is the polarization of a single dipole, $\rho\left(v_{c}{ }^{+}, v_{c}{ }^{-}\right)$the density function of the dipoles with respect to their individual coercive voltages and $D$ a direction operator \pm 1 depending on the actual voltage and the turning points it has passed. The tanh-function is a useful approximation for the integral in (1) when $\rho$ is taken as a Gaussian distribution:

$$
P\left(V_{\text {ext }}\right)=F \cdot P_{\text {sat }} \cdot \tanh \left[a\left(V_{\text {ext }} \mp V_{C}^{ \pm}\right)\right]
$$

Its derivative is used for the parameter extraction:

$$
\mathrm{d} P / \mathrm{d} V\left(V_{\text {ext }}\right)=F \cdot P_{\text {sat }} \cdot a / \cosh ^{2}\left[a\left(V_{\text {ext }} \mp V_{C}{ }^{ \pm}\right)\right]
$$

$P_{\text {sat }}$ is the saturation polarization and $V_{C}^{ \pm}$is the macroscopic coercive voltage, i.e. the mean value of the individual coercive voltages. The behavior of the non-saturation loops are taken into account by a factor $F$, so that $F \cdot P_{\text {sat }}$ represents that proportion of dipoles that take part in the switching process of the nonsaturation loop. This model [10] describes the initial dipole distribution before fatigue takes place (fig.2a). A model including time dependent phenomena is described in 11, 12].

\section{Modeling FATigue}

We assume that, e.g., due to charged interface traps, some dipoles underlie a change of their individual coercive force during cycling. The impact of this mechanism, however, can vary strongly with the ferroelectric and electrode materials and the manufacturing process. In each cycle some dipoles get stuck and loose their ability to switch. In the case of low-field fatigue it can be restored by a poling cycle, i.e. a voltage is applied, that is high enough to eliminate interface charges and thus re-mobilizing the pinned dipoles.

The dipoles in the regions close to the interface get a preferential polarization, which means that they easily turn into one direction, but need a higher voltage to switch to the opposite. This is valid for the opposite electrode as well, resulting in a certain amount of dipoles which favor the opposite polarization.

Thus, with an increasing number of cycles the original probability distribution of the dipoles with respect to their individual coercive forces undergoes
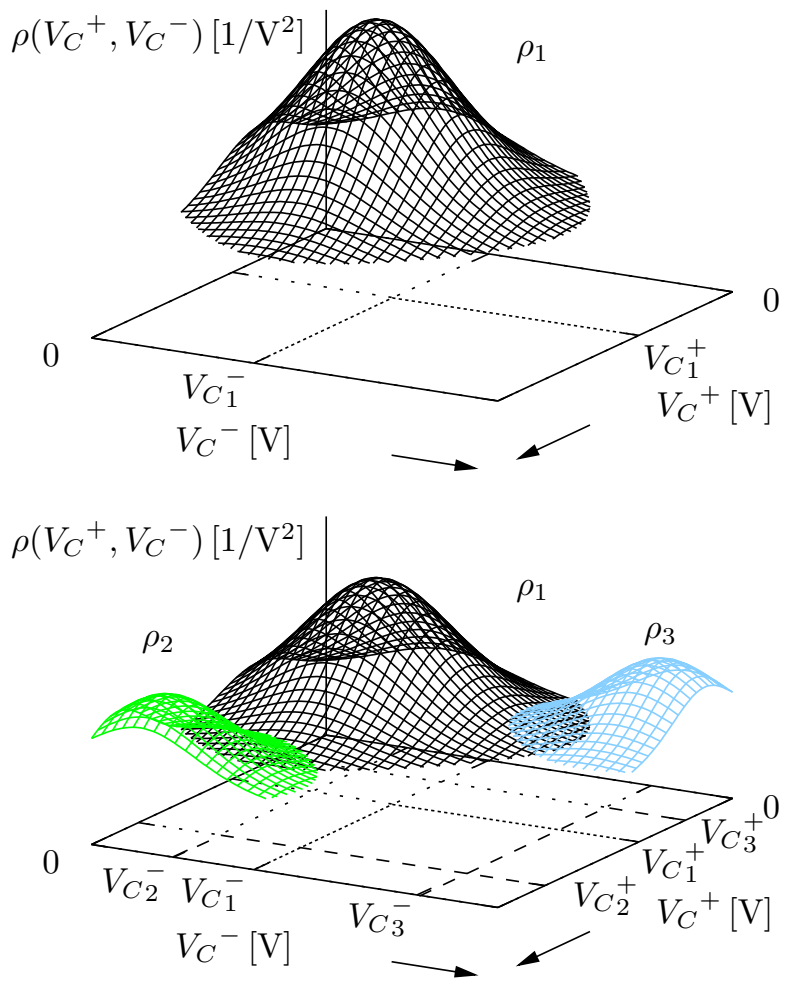

Fig. 2: a) Probability density function before fatigue b) Probability density function after fatigue

some changes. The amount of dipoles that are not affected by interface charges decreases, resulting in a Gaussian distribution with a lower $P_{\text {sat }}$. The preferential polarization of the dipoles affected by the interface traps is modeled by an additional probability distribution with a lower $V_{C}{ }^{+}$in the preferred direction and a higher $V_{C}{ }^{-}$in the opposite direction. A third probability distribution exists for the dipoles with preference of the opposite direction. This results in a total probability distribution $\rho\left(v_{c}{ }^{+}, v_{c}{ }^{-}\right)$that consists now of three Gaussian peaks (fig.2 $\mathrm{b}$ ). For the total polarization the following equation is valid:

$$
P\left(V_{\text {ext }}\right)=\sum_{i=1}^{3} F_{i} \cdot P_{\text {sat } i} \cdot \tanh \left[a_{i}\left(V_{\text {ext }} \mp V_{C_{i}^{ \pm}}^{ \pm}\right)\right]
$$

The factors $F_{1,2,3}$ are calculated with the help of the Preisach model, but for each Gaussian peak separately. Therefore, in the simulation the ferroelectric capacitor is modeled by three parallel ferroelectric capacitors, in order to account for the minor loops of the hysteresis.

\section{Measurement and Parameter Extraction}

Fig.3 shows the simulated derivative $\mathrm{d} P / \mathrm{d} V$ of the hysteresis curve for a fresh capacitor and a fatigued one.

From (4) $\mathrm{d} P / \mathrm{d} V_{\text {ext }}$ is derived for the case of a fatigued 


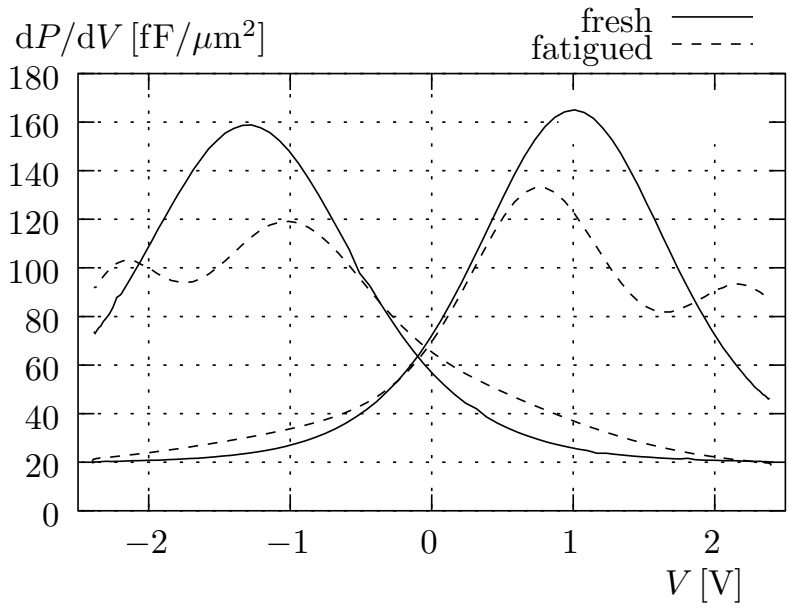

Fig. 3: Simulated fatigue behavior of capacitance characteristic $\mathrm{d} P / \mathrm{d} V$.

ferroelectric capacitor:

$$
\frac{\mathrm{d} P}{\mathrm{~d} V_{e x t}}=\sum_{i=1}^{3} \frac{F_{i}^{ \pm} \cdot P_{\text {sat } i} \cdot a_{i}^{ \pm}}{\cosh ^{2}\left[a_{i}^{ \pm}\left(V_{e x t}-V_{C_{i}^{ \pm}}\right)\right]}
$$

The + is valid for increasing $V_{\text {ext }}$ and the - for decreasing $V_{\text {ext }}$. Here the main peaks $1^{ \pm}$represent the set of undisturbed dipoles, whereas the adjacent peaks $2^{ \pm}$and $3^{ \pm}$represent the set of dipoles that have a preferential orientation. In fig.4 the $\mathrm{d} P / \mathrm{d} V$ curve is shown for both increasing and decreasing $V_{e x t}$ which depicts the switching behavior of the dipoles.

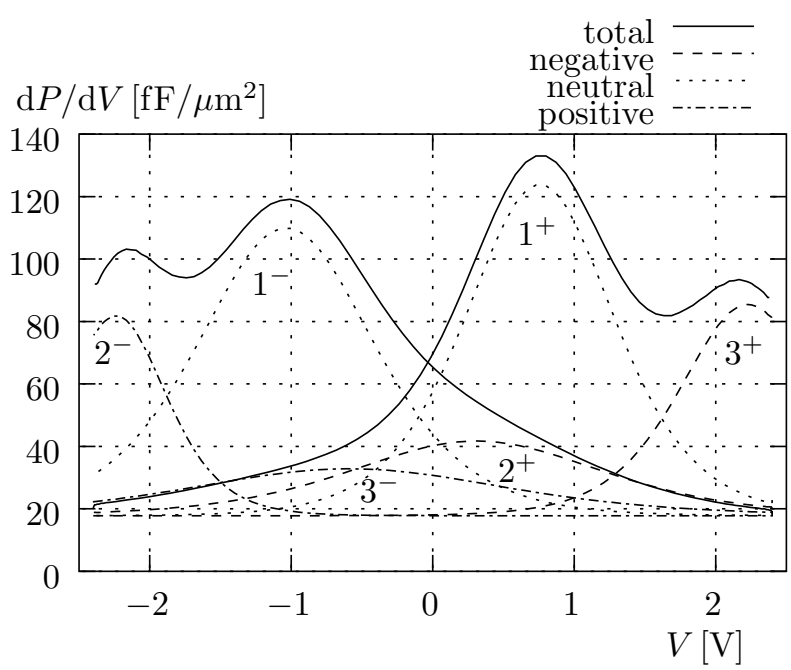

Fig. 4: Simulated fatigue capacitance characteristic $\mathrm{d} P / \mathrm{d} V$ consisting of three Gaussian peaks.

Each of the three sets of dipoles causes a peak for each direction of $V_{\text {ext }}$, e.g., the peak farthest on the left for the decreasing voltage has its correspondent on the farthest left for the increasing voltage $\left(2^{-} \hat{=} 2^{+}\right)$. This means that this set of dipoles is biased to positive po- larization. This statement is also valid for the negatively biased set of dipoles. In order to use formula (5) for the extraction of the necessary parameters, it has to be taken into account that due to the high $\left|V_{C_{3}^{+}}^{+}\right|$ and $\left|V_{C_{2}}^{-}\right|$the applied voltage may not be sufficient to drive the peaks $3^{+}$and $2^{-}$into full saturation. This means that for the contrary voltage gradient the factors $F_{2}^{+}$and $F_{3}^{-}$do not equal 1 as they represent that fraction of dipoles that takes part in switching. Their calculation follows:

$$
F_{2}^{+}=\frac{2 P_{\text {sat } 2}-A}{2 P_{\text {sat } 2}}
$$

The amount of dipoles $A$ do not take part in the switching as their individual coercive voltages $v_{c}{ }^{-}$are beyond the turning point $V_{\min }$ of the applied voltage. With $2 P_{\text {sat } 2}$ representing the change of polarization for the whole amount of dipoles in peak $2^{-}, A$ follows directly from (4) and (6):

$$
\begin{gathered}
A=P_{\text {sat } 2} \cdot\left(1+\tanh \left[a_{2}^{-} \cdot\left(V_{\text {min }}-V_{C_{2}^{-}}\right)\right]\right) \\
F_{2}^{+}=\frac{1-\tanh \left[a_{2}^{-} \cdot\left(V_{\min }-V_{C_{2}}^{-}\right)\right]}{2}
\end{gathered}
$$

The calculation for $F_{3}^{-}$achieves a similar result:

$$
F_{3}^{-}=\frac{1+\tanh \left[a_{3}^{-} \cdot\left(V_{\max }-V_{C_{3}^{+}}^{+}\right)\right]}{2}
$$

Fig. 5. shows the result of our parameter extraction for the negative voltage gradient.

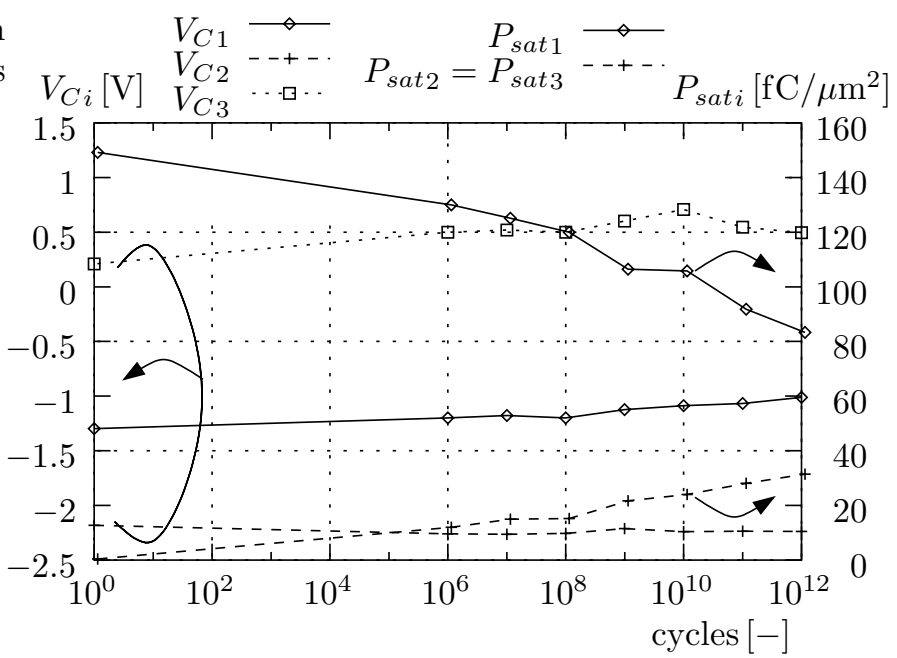

Fig. 5: Extracted parameters $V_{C i}$ and $P_{s a t i}$ for the negative voltage gradient.

While extracting the parameters one has to be aware that especially for the measurement curves where the adjacent peaks are less pronounced, a certain ambiguity exists in dividing the total $\mathrm{d} P / \mathrm{d} V$ curve into the three peaks. Fig.6 shows the measurement as taken from [5] and the simulated curves resulting from the extracted parameters. Fig. [7 compares the measured and simulated hysteresis for a fresh ferroelectric cell as well as for a $10^{9}$ cycles fatigued one. 


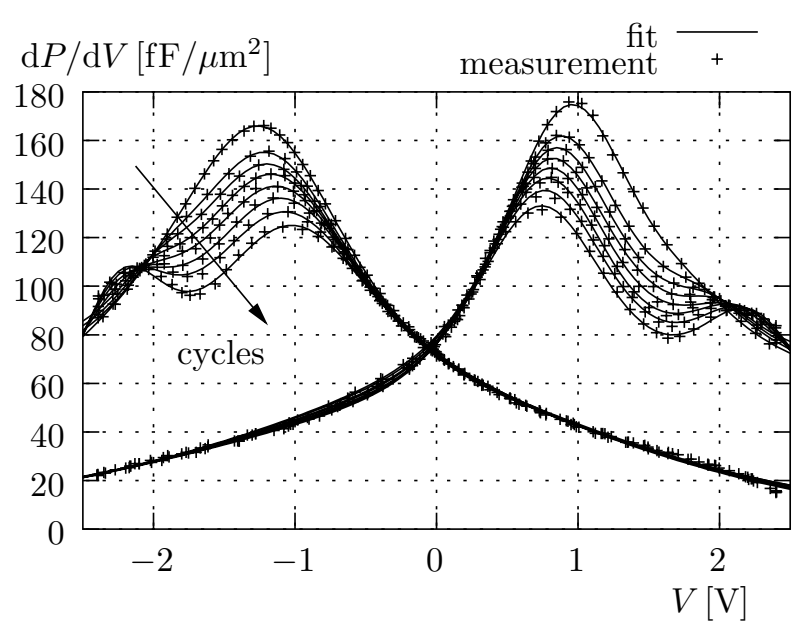

Fig. 6: Fatigue behavior of capacitance characteristic $\mathrm{d} P / \mathrm{d} V$. Comparison between measurement and model simulation for different number of cycles.

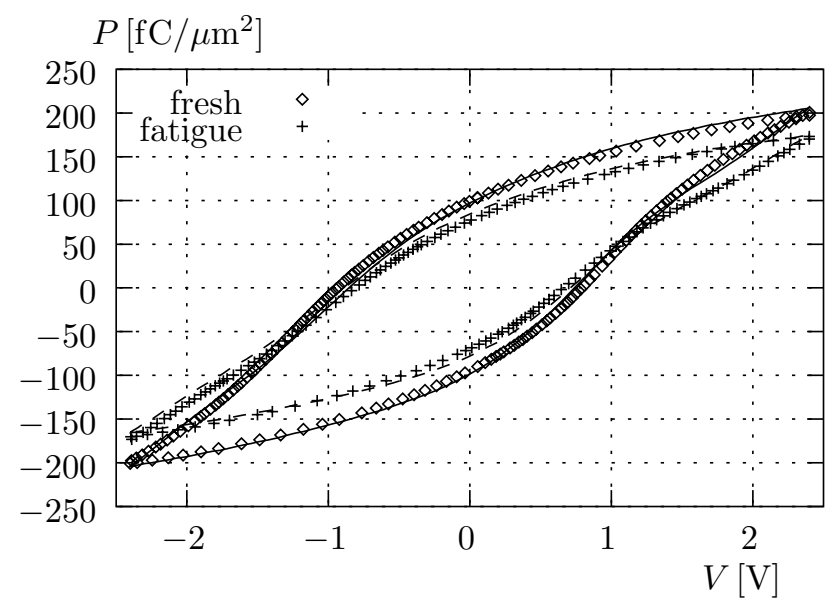

Fig. 7: Measured (symbols) and simulated (lines) hysteresis loop for a fresh and fatigued $\left(10^{9}\right.$ polarization cycles) cell.

\section{Circuit Simulation}

With our new model predictions for FRAM cells in memory arrays can be performed.

Fig.8 shows a $1 \mu \mathrm{m}^{2}$ FRAM cell with a transfer transistor, controlled by a word line WL, and a bit line $\mathrm{BL}$ with a parasitic capacitance of $100 \mathrm{fF}$. The plate of the cell is held on a constant voltage of $1.5 \mathrm{~V}$, while the states "0" or "1" are written by pulsing the bit line. The cell is evaluated by precharging the bit line to $0 \mathrm{~V}$ and subsequent discharging of the FRAM cell. Simulating this circuit for a fresh and a $10^{9}$ cycled fatigue cell results in a diminution of the signal-to-noise-ratio of $33 \%$.

This has to be taken into consideration for accurate designing of memory arrays.

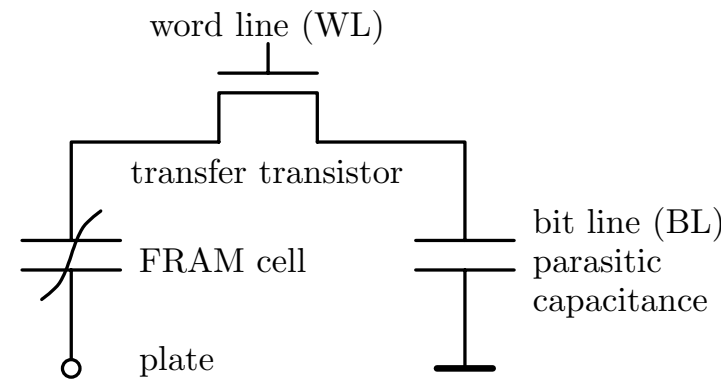

Fig. 8: Equivalent circuit for a memory cell.

\section{CONCLUSION}

The observed increase of coercitive voltage dependent on the number of cycles at low field fatigue can be modeled by a fatigue compact model. The model is an improvement of the Preisach model that is refined by introducing a parallel structure of three ferroelectric capacitors with different coercitive voltages.

An extraction method for the necessary parameters is presented. The characterized hysteresis corresponds to the measured one. The simulation of FRAM cell arrays shows a reduction for a $10^{9}$ cycled fatigue of $33 \%$. The fatigue characteristics of ferroelectric materials are noticeably influenced by the processing and patterning technique for capacitors.

This model can easily be adapted to changed fatigue characteristics.

\section{REFERENCES}

[1] O. Auciello. Integr. Ferroelectr., 15:211, 1997.

[2] H. Watanabe et al. In Proc. of $4^{\text {th }}$ ISIF, pages 346-362, Monterey, March 1992.

[3] H. Al-Shareef et al. J. Appl. Phys., 77:2146, 1995.

[4] C. A. Paz de Araujo et al. Nature, 374:627, 1995.

[5] R. Moazzami. Ferroelectric thin film technology for semiconductor memory. Semicond. Sci. Technol., 10:375-390, 1995.

[6] D. Dimos et al. J. Appl. Phys., 80:1682, 1996.

[7] J. J. Lee et al. J. Appl. Phys., 78:5073, 1995.

[8] T. Mihara et al. Jpn. J. Appl. Phys., 33:3996, 1994.

[9] P. K. Larsen et al. J. Appl. Phys., 76:2405, 1994.

[10] B. Jiang et al. Computationally efficient ferroelectric capacitor model for circuit simulation. In Symp. on VLSI Technology, pages 141-142, 1997.

[11] C. Kühn et al. A dynamic ferroelectric capacitance model for circuit simulators. In ISAF'2000.

[12] C. Kühn et al. A new physics model for the relaxation in ferroelectrics. In ESSDERC'2000. 